\title{
A New Way to Approximate Sine
}

\section{Rimpu Ray}

\begin{abstract}
I discuss about a new way to approximate sine, in degree measurement from $15^{\circ}$ to $90^{\circ}$. Formula is divided into some parts.

Index Terms-About four key words or phrases in alphabetical order, separated by commas.
\end{abstract}

\section{INTRODUCTION}

The motivation of this paper is the sine approximation formula of Bhaskara 1(c. 600- c.680).He was an Indian mathematician. I want to say Bhaskara 1 's sine approximation formula as THE FATHER OF SINE APPROXIMATION. This formula is still unknown to many people. The formula had been given in his treatise titled MAHABHASKARIYA. It is not known how he derived this formula. The main formula is $\sin x^{\circ}=\frac{4 x(180-x)}{40500-x(180-x)} ; x €$ $\left[0^{\circ}, 180^{\circ}\right]$. Mathematicians have given many approaches to derive this formula. The radian measure of sine was also given in his treatise. We can also approximate sine by "taylor series" and many other methods.

I try to describe one new method on this paper. The formula is divided into 3 parts, which is given by.

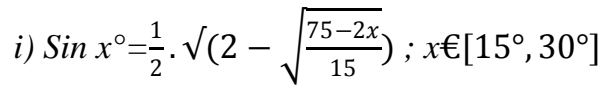

ii) $\operatorname{Sin} x^{\circ}=\frac{1}{2} \cdot \sqrt{(}\left(\frac{X-15}{15}\right) ; x €\left[30^{\circ}, 60^{\circ}\right]$.

ii) $\operatorname{Sin} x^{\circ}=\frac{1}{60} \cdot \sqrt{ }\{(x-30) \cdot(150-x)\} ; x €\left[60^{\circ}, 90^{\circ}\right]$.

\section{MY ALGEBRAIC APPROACH TO APPROXIMATE SINE}

We know that for isosceles triangle,

$3^{\text {rd }}$ side $=2$. (Measure of any of the equal sides of the isosceles triangle). $\sin \left(\frac{\alpha}{2}\right)$; where $\alpha$ is the angle between two equal sides of the isosceles triangle.

When $\alpha=60^{\circ}$, we get

(Measure of any of the equal sides of the isosceles triangle) $\sqrt{1}=2$. (Measure of any of the equal sides of the isosceles triangle). $\operatorname{Sin}\left(\frac{60^{\circ}}{2}\right)$ -

Again, for right angle isosceles triangle i.e. when $\alpha=90^{\circ}$, using 1 we get,

(Measure of any of the equal sides of the isosceles triangle). $\sqrt{2}=2$. (Measure of any of the equal sides of the isosceles triangle). $\sin \left(\frac{90^{\circ}}{2}\right)$ (1.1)

Rimpu Ray, Mathematics Department, Gauhati University (Guwahati, Assam, India)
Again for $\alpha=120^{\circ}$, we get

(Measure of any of the equal sides of the isosceles triangle). $\sqrt{3}=2$. (Measure of any of the equal sides of the isosceles triangle). Sin $\left(\frac{120^{\circ}}{2}\right)$ (1.3)

So from 1.1, 1.2 and 1.3 we see that,

$\sqrt{c}=2 \cdot \sin \left(\frac{\alpha}{2}\right) ; c €[1,3]$ and $\alpha €\left[60^{\circ}, 120^{\circ}\right]$.

Now, we have to find the value of $c$. Since the value of $c$ starts from 1 and ends at 3 with respect to change in $\alpha$, which starts from $60^{\circ}$ and ends at $120^{\circ}$.

Therefore, $c=1+\frac{\alpha-60}{30}$

$$
\Rightarrow c=\frac{\alpha-30}{30}
$$

Now from (2) we have

$\sqrt{\left(\frac{\alpha-30}{30}\right)}=2 \cdot \sin \left(\frac{\alpha}{2}\right) ; \alpha €\left[60^{\circ}, 120^{\circ}\right]$

Taking $\alpha=2 x$, we can rewrite the equation as

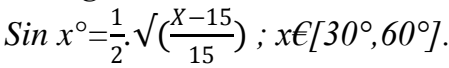

Again we know that,

$\operatorname{Sin} x=2 \cdot \sin \left(\frac{x}{2}\right) \cdot \cos \left(\frac{x}{2}\right)$

From 3 we have,

$\left.\operatorname{Sin}\left(\frac{x}{2}\right)=\frac{1}{2} \cdot \sqrt{\left(\frac{x}{2}-15\right.} \frac{15}{15}\right)$

And $\left.\operatorname{Cos}\left(\frac{x}{2}\right)=\sqrt{ }\left[1-\left\{\frac{1}{2} \cdot \sqrt{\left(\frac{x}{2}-15\right.} \frac{15}{15}\right)\right\}^{2}\right]$

So putting 4.1 and 4.2 in 4 we have,

$\operatorname{Sin} x^{\circ}=\frac{1}{60} \cdot \sqrt{ }\{(x-30) .(150-x)\} ; x €\left[60^{\circ}, 120^{\circ}\right]$.

Or we can say,

$\operatorname{Sin} x^{\circ}=\frac{1}{60} \cdot \sqrt{ }\{(x-30) .(150-x)\} ; x €\left[60^{\circ}, 90^{\circ}\right]$.

Again, we know that

$\operatorname{Sin}\left(\frac{x}{2}\right)=\sqrt{(}\left(\frac{1-\cos x}{2}\right)$

Now using 3 in $\sin ^{2} x+\cos ^{2} x=1$ we have

$$
\operatorname{Cos} x=\sqrt{(}\left(\frac{75-x}{15}\right)
$$

Using 5.1 in 5 we can derive the following formula,

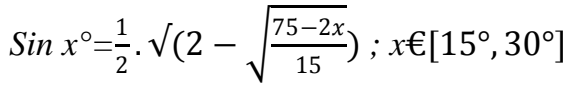

\section{ACCURACY}

Let's talk about the accuracy of the formula with some examples.

In real, $\sin 20^{\circ}=0.34202014332$

My formula gives the result $\sin 20^{\circ}=$ 0.3436840007332317 .

Again $\sin 50^{\circ}=0.766044443118978$.

According to my formula it gives $\sin 50^{\circ}=0.76376261528$.

Similarly, let's check for $75^{\circ}$

$\operatorname{Sin} 75^{\circ}=0.96592582628$.

But, my formula gives $\sin 75^{\circ}=0.96824583655$. 
A New Way to Approximate Sine

As we can see above the error is negligible. So the formula is quite accurate according to the exam purpose and for other use also.

\section{CONCLUSION}

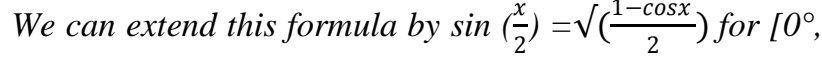
$15^{\circ} \mathrm{J}$, in many parts as I have mentioned above. We can also find the $\cos x$, tan $x$ and other trigonometric identities by this formula.

\section{REFERENCES}

[1] R.C. Gupta (1967). "Bhaskara I' approximation to sine". Indian Journal of HIstory of Science. Archived from the original on 16 March 2012. Retrieved 20 April 2010 